\title{
Laboratory evaluation of the effect of unfilled resin after the use of self-etch and total-etch dentin adhesives on the Shear Bond Strength of composite to dentin
}

\author{
Ehsan Baradaran Nasseri ${ }^{1}$, Sara Majidinia ${ }^{2}$, Davood Aghasizadeh Sharbaf ${ }^{3}$
}

${ }^{1}$ DDs, MS. Assistant Professor, Department Of Operative Dentistry, School of Dentistry, Mashhad University Of Medical Sciences, Mashhad, Iran

${ }^{2}$ DDs, MS. Assistant Professor, Dental Materials Research Center, Mashhad University of Medical Sciences, Mashhad, Iran

${ }^{3}$ DDs, MS. Assistant Professor, Oral and Maxillofacial Disease Research Center, School of Dentistry, Mashhad University Of Medical Sciences, Mashhad, Iran

\section{Type of article: Original}

\begin{abstract}
Background: Based on the frequent application of composite resins as tooth-colored fillings, this method is considered a viable alternative to dental amalgam. However, this method has the low bond strength of the composite to dentin. To solve this issue, various dental adhesive systems with different characteristics have been developed by dentistry experts.

Aim: To assess the effect of an additional layer of unfilled resin in self-etch and total-etch dentin adhesives on the shear bond strength (SBS) of composite to dentin. Moreover, we assessed the effects of sample storage in artificial saliva on the SBS of composite to dentin.

Methods: Methods: This experimental study was conducted on 160 freshly extracted human first or second premolar teeth, which were randomly divided into 16 groups. The teeth were prepared from Mashhad University of Medical Sciences, Mashhad, Iran (2008-2009). Scotchbond Multi-purpose (SBMP), single bond (SB), Clearfil SE Bond, and Clearfil S3 Bond were applied to dentin surface with or without the placement of hydrophobic resin (Margin Bond) in accordance with the instructions of the manufacturers. To expose the coronal dentin, the teeth were abraded with 600 grit $\mathrm{SiC}$ paper. Immediately after restoration, half of the samples were tested in terms of SBS, while the other samples were evaluated in terms of SBS after three months of storage in artificial saliva. SBS rates of dental composites evaluated by universal testing machine and samples were studied by optical stereomicroscopy to verify the failure type. Data analysis was performed in SPSS V.16 using Kolmogorov-Smirnov test, independent-samples t-test, ANOVA, and Duncan's logistic regression test.

Results: In this study, a significant reduction was observed in the SBS rates of SB and S3 bond adhesive systems after storage with and without hydrophobic resin $(p>0.000)$. Without storage in normal saline, a significant increase was observed in the SBS rate of the SE bond $(\mathrm{p}=0.013)$. In addition, SBS rate of SBMP significantly increased after storage with hydrophobic resin $(\mathrm{p}=0.001)$. Finally, the highest and lowest rates of SBS were observed in the SE and S3 bonds in all the experimental groups, respectively.

Conclusion: The effects of using a hydrophobic resin layer on shear bond strength values seem to be effective. The mild self-etch adhesive exhibited the best resin-dentine bond strength after aging

Keywords: Unfilled resin, Dentin adhesives, Shear bond strength, Composite
\end{abstract}

\section{Introduction}

Tooth-colored fillings are used to cosmetically change the size, color and shape of the teeth. Due to the frequent application of composite resins as tooth-colored fillings, this method is considered a viable alternative to dental

\section{Corresponding author:}

Davood Aghasizadeh Sharbaf, Department of Prosthodontic, Oral and Maxillofacial Disease Research Center, School of Dentistry, Mashhad University of Medical Sciences, Mashhad Iran.

Tel: +989153130534, Fax: +985138414499, Email: aghasizadehd@mums.ac.ir

Received: July 10, 2016, Accepted: December 24, 2016, Published: May 2017

iThenticate screening: October 02, 2016, English editing: March 12, 2017, Quality control: March 27, 2017

(C) 2017 The Authors. This is an open access article under the terms of the Creative Commons Attribution-NonCommercialNoDerivs License, which permits use and distribution in any medium, provided the original work is properly cited, the use is non-commercial and no modifications or adaptations are made. 
amalgam (1). Regarding the numerous use of composite resins as tooth-colored fillings, this method is considered a worthwhile substitute to dental amalgam. Nevertheless, this is the low bond strength of the composite to dentin, particularly when used on the dentin tissue. Subsequently, several dental adhesive methods with diverse characteristics have been emerged by dentistry researchers. As a reconstructive technique, composite resin is widely used for dental restoration; however, this technique has been associated with several problems. Such example is the low bond strength of the composite to dentin, especially the dentin tissue. To solve this issue, various dental adhesive systems with different characteristics have been developed by dentistry experts $(2,3)$. Discovery of different generations of adhesives has resulted in increased shear bond strength (SBS) of composite to dentin. Regardless of the adhesive type, the immediate bond strengths of the majority of adhesive systems have been shown to be appropriate. However, clinical application of these adhesives may lead to the reduced rate of bond strength in some adhesives, while other adhesive types are reported to have greater resistance. In other words, durability of the bond strength of adhesives mainly depends on the maintenance of the adhesive system $(4,5)$. Several techniques are used to determine bond strength, including shear and tensile bond strength. As is known, extra adhesion between the teeth and adhesives is associated with higher resistance to the stress induced by resin polymerization and natural functional forces. Preservation of dental samples in vitro is performed using various techniques, the most common of which involves storage in water, artificial saliva or an incubator (6). According to the literature, bond strength significantly decreases following the storage of samples in water or artificial saliva even after a short time, which is probably due to the hydrolytic degradation of adhesive components. On the same note, water plasticization may diminish the mechanical properties of polymer-matrix composites through reducing the frictional forces between the polymer chains $(5,7)$. Use of adhesive systems is associated with numerous changes to improve the binding properties of these compounds. For instance, an additional adhesive layer or a hydrophobic resin layer are commonly applied in bonding so as to create a thick intermediate layer with low elasticity between the tooth and composite for the absorption of the stress induced by composite shrinkage, which leads to the enhancement of SBS (8). In recent years, use of dental adhesives has advanced remarkably, and enamel etching with phosphoric acid has been shown to improve enamel adhesion (8). According to a study by Moazami et al., addition of a hydrophobic layer to self-etch adhesives significantly decreased gingival microleakage. Therefore, it was inferred that the placement of a hydrophobic resin layer following the application of adhesives could form a hydrophobic, elastic layer and reduce or eliminate permeability (9). This study was conducted to determine the effect of hydrophobic resin on the SBS of composite to dentin in different conditions following the application of various adhesive systems. Furthermore, we aimed to determine the effect of an additional layer of unfilled resin with self-etch and total-etch dentin adhesives on the SBS of composite to dentin. Also, we assessed the effect of sample storage in artificial saliva on the SBS of composite to dentin and verified the possible relationship between the mode of graft failure and SBS rate.

\section{Material and Methods}

\subsection{Research design and sample preparation}

This experimental study was conducted on 160 freshly extracted human first or second premolar teeth, which had been removed due to orthodontic causes. The teeth were prepared from Mashhad University of Medical Sciences, Mashhad, Iran (2008-2009). Sample size was determined based on a pilot study by Moazami et al. performed in 2007 (3). All the selected teeth were healthy and had no caries, and normal saline was used for the preservation of these samples. Initially, the samples were cleaned using a Cavitron device with pumice slurry and water in order to eliminate tissue redundancies. Afterwards, the teeth were closely examined using a magnifying glass, and samples with any cracks were excluded from further evaluation. In the next stage, the teeth enamel (occlusal surface) was removed using a diamond disk (D\&Z, Darmstadt, Germany) and water spray. Occlusal dentin surfaces were abraded using wet 600-grit sandpaper in order to prepare the samples for composite connection. Following that, the teeth were divided into four experimental groups. Each sample (length: $20 \mathrm{~mm}$, diameter: $20 \mathrm{~mm}$ ) was placed in cylindrical polyvinyl chloride and adjusted by temporary acrylic.

\subsection{Experimental groups}

Collected samples were randomly divided into 16 groups of 10, as follows:

1) Group one: In samples of this group, Scotchbond Etchant (35\% phosphoric acid colloidal silica) total-etch 3 -stage adhesive system was used. Enamel and dentin were etched for 15 seconds, rinsed with water for 15 seconds, and dried for 5 seconds. Afterwards, Scotchbond Multi-purpose (SBMP) adhesive (3M, USA) was used without hydrophobic resin (Margin Bond) according to the instructions of the manufacturer. After composite restoration (which was explained later) were completed, the samples were artificial saliva aged (ASA) for three months. 
2) Group two: In this group, Scotchbond Etchant (35\% phosphoric acid colloidal silica) total-etch 2-stage adhesive system was used. Samples were etched for 15 seconds, rinsed with water for 15 seconds, and dried for 5 seconds. Afterwards, single bond (SB) adhesive (3M, USA) was applied without hydrophobic resin according to the instructions of the manufacturer. After composite restoration (which was explained later) were completed, the samples were artificial saliva aged (ASA) for three months.

3) Group three: In this group, we used the Clearfil SE Bond (SEB) (Kuraray, Japan) self-etch 2-stage adhesive system. After the application of primer to the samples for 20 seconds, the samples were dried by mild airstream. Following that, the bonding was brushed on cavity surfaces, which were exposed to air and treated for 10 seconds without hydrophobic resin according to the instructions of the manufacturer. After composite restoration (which was explained later) were completed, the samples were artificial saliva aged (ASA) for three months

4) Group four: In this group, we used the Clearfil S3 Bond (S3B) all-in-one self-etch adhesive system (Kuraray, Japan). The bonding was applied to cavity surfaces using a brush for 20 seconds. Afterwards, the samples were exposed to air and treated without hydrophobic resin according to the instructions of the manufacturer. After composite restoration (which was explained later) were completed, the samples were artificial saliva aged (ASA) for three months.

The same adhesive systems were applied in groups 5-8, with the exception of an additional layer of hydrophobic unfilled resin (Margin Bond) (Coltene, Swiss), the restorative material of which was air-thinned and light-cured (without ASA). In this study, all the products were used in accordance with the instructions of the manufacturers. Furthermore, the same adhesives were applied in groups 9-12, with the exception of the application of ASA for three months (without margin bond). In groups 13-16, the same adhesives were used, in addition to a hydrophobic unfilled resin layer and ASA for three months.

\subsection{Technique of sample preparation}

Bonding systems were applied to the samples after preparation. Afterwards, special mold (depth: $2 \mathrm{~mm}$, diameter: 1 $\mathrm{mm}$ ) were used for the bonding composite to the dentin surface. Moreover, the composite resin Z100 (3M ESPE) shade A2 was bonded in layers to the samples and cured for 40 seconds by Astralis 7 (Ivoclar Vivadent) with light intensity of 700 milliwatts per square centimeter using the low-power program.

\subsection{Measurement of shear bond strength (SBS)}

After restoration, half of the samples were immediately evaluated in terms of SBS, while the remaining samples were assessed in terms of SBS after three months of storage in artificial saliva in an incubator (at temperature of 37 ${ }^{\circ} \mathrm{C}$ with 100\% humidity). SBS was evaluated using Zwick Z250 (Germany) (load cell: 2,000 Newton, preload: 5 Newton, force: 0.5 millimeters per minute). Finally, the dental composites and dental samples were collected and studied using an optical stereomicroscopy (Blue Light Industry, La Habra LA, USA) at magnifications of 20X and 40X to determine the mode of graft failure. In addition, a digital camera (Exwave HAD, Sony, Tokyo, Japan) was used to visually record the type of failure of samples. In this study, type of failure was classified into three modes of adhesive, cohesive, and mixed.

\subsection{Data analysis}

Data analysis was performed in SPSS version 16 (SPSS inc., Chicago, Illinois, USA) using Kolmogorov-Smirnov test, independent-samples t-test, ANOVA, and Duncan's logistic regression test, and $\mathrm{p}$ value of less than 0.05 was considered significant.

\section{Results}

Evaluation of the effect of ASA application on the SBMP adhesive without a hydrophobic resin layer for three months indicated that use of ASA caused a significant decrease in the SBS rate of the SBMP adhesive system $(\mathrm{t}=4.057)(\mathrm{p}=0.001)$. Our findings were indicative of a significant reduction in the SBS rate of the SB adhesive system after the application of ASA without a hydrophobic resin layer $(\mathrm{t}=10.507)(\mathrm{p}<0.0005)$. Moreover, a significant reduction was observed in the SBS rate of the S3B adhesive system without a hydrophobic resin layer $(\mathrm{t}=9.584)(\mathrm{p}<0.0005)$. However, assessment of the SBS rate of the SEB adhesive system without a hydrophobic resin layer showed that application of ASA slightly decreased the rate of SBS in the SEB adhesive system ( $\mathrm{t}=0.015)$ $(\mathrm{p}=0.988)$. In this study, we evaluated the effect of ASA application with a hydrophobic resin layer on different adhesives within three months. Our findings in this regard were indicative of an insignificant decrease in the SBS rate of the SBMP adhesive system $(\mathrm{t}=2.009)(\mathrm{p}=0.06)$. In addition, no significant reduction was observed in the SBS 
rate of the SEB adhesive system $(\mathrm{t}=1.514)(\mathrm{p}=0.147)$. There was a significant reduction in the SBS rate of the SB adhesive system using ASA with an additional hydrophobic resin layer $(t=9.802)(p<0.0005)$. Moreover, the SBS rate of the S3B adhesive system significantly reduced following the application of ASA with a hydrophobic resin layer $(t=7.078)(p<0.0005)$. Assessment of the effect of the hydrophobic resin layer without ASA in the first and second four groups is presented in Figure 1. According to the results of this study, the rate of SBS was higher in the SBMP and SEB adhesive systems with an additional hydrophobic resin layer (with or without the application of ASA). Moreover, the rate of SBS was higher in the SB and S3B adhesive systems without the application of ASA (with or without hydrophobic resin). Investigation of different adhesive systems in the first and second four groups was indicative of no significant difference in the first four groups without the application of hydrophobic resin and ASA $(\mathrm{f}=2.325)(\mathrm{p}=0.091)$. However, in the second four groups, a significant difference was observed between the adhesive systems with the application of hydrophobic resin layer and ASA $(f=16.384)(p<0.005)$. Results of this study were indicative of no significant difference between the SBMP and SEB adhesive systems $(\mathrm{p}=0.145)$; however, there was a significant difference in other adhesive types in this regard (third four groups and fourth four groups) $(\mathrm{p}<0.005)$. Accordingly, the highest and lowest SBS rates were reported in the SEB and S3B adhesive systems, respectively. Assessment of different adhesives in groups 9-12 was indicative of a significant difference with the application of ASA without the use of hydrophobic resin (groups 9-12) $(f=58.673)(p<0.0005)$. In addition, a significant difference was observed in different adhesive systems in groups 9-12 ( $\mathrm{f}=58.673)(\mathrm{p}<0.0005)$. In this regard, a significant difference was observed between different adhesives with the application of hydrophobic resin and ASA (groups 13-16) $(\mathrm{f}=84.598)(\mathrm{p}<0.005)$.

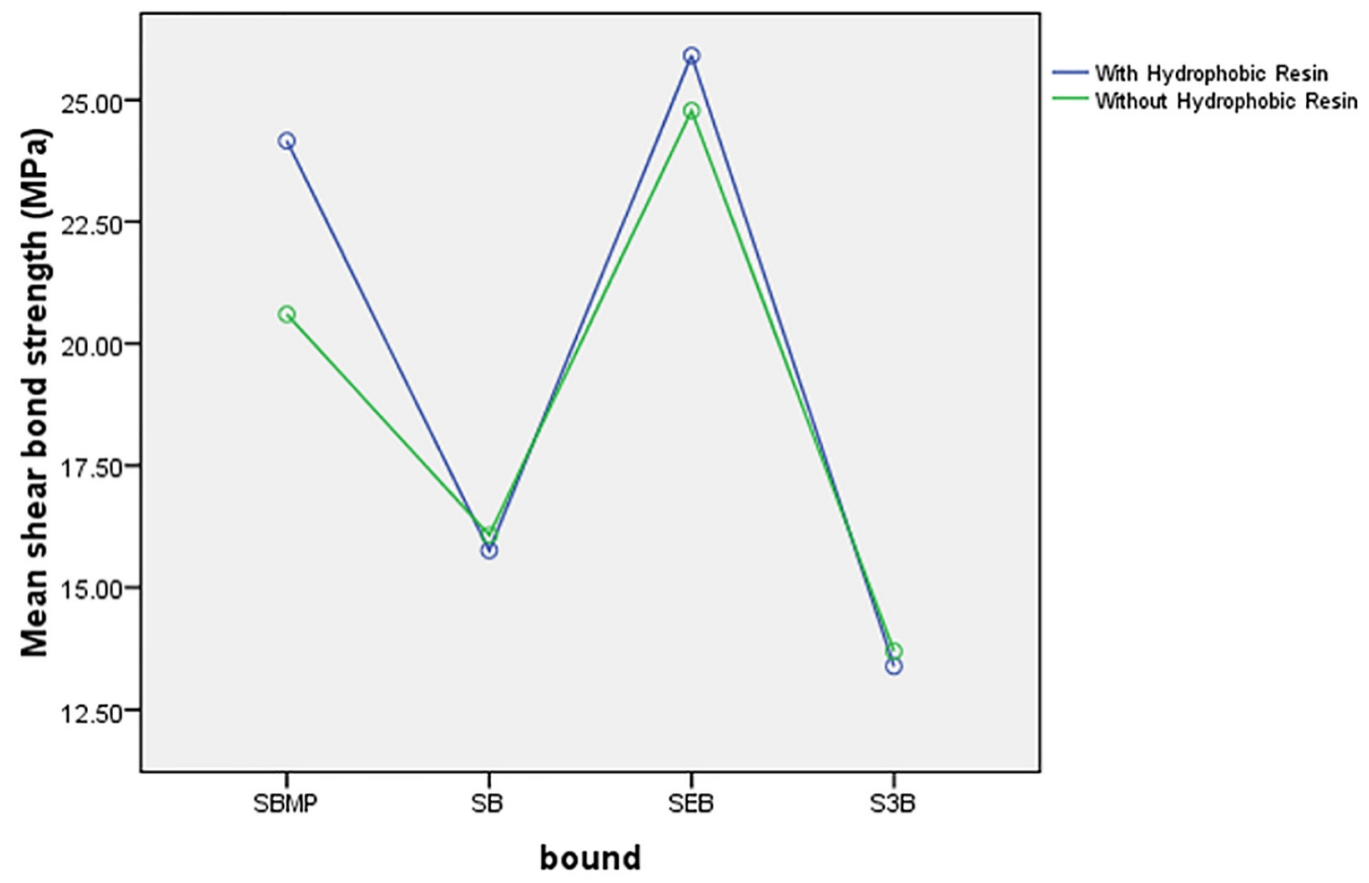

Figure 1. Mean of shear bond strength (SBS) (MPa) in experimental groups 9-16 without artificial saliva aging (ASA)

There was no significant difference between the SBMP and SEB adhesive systems in terms of SBS ( $\mathrm{p}=0.73$ ), while a significant difference was found between other types of adhesives in this regard $(p<0.005)$. The highest and lowest SBS rates were observed in SEB and S3B adhesives, respectively. In this study, we compared the effects of hydrophobic resin and ASA in total-etch (SBMP and SB) and self-etch adhesives (SEB and S3B) on the rate of SBS. According to the findings, there was no significant difference between total-etch and self-etch adhesives in this regard $(\mathrm{p}=0.703)$. According to the information in Diagram 3, use of hydrophobic resin increased the rate of SBS in both total-etch and self-etch adhesives. Furthermore, the rate of SBS was found to be higher in total-etch adhesives compared to self-etch adhesives with or without hydrophobic resin. Also, use of hydrophobic resin increased the SBS rate in all experimental groups with ASA application. In both total-etch and self-etch adhesives with and without the use of hydrophobic resin, the SBS rate was observed to be lower with the application of ASA. In totaletch adhesives with and without hydrophobic resin, the rate of SBS was lower with the application of ASA. 
Additionally, the rate of SBS in self-etch adhesives with and without hydrophobic resin was lower with the application of ASA (Figure 2). Findings of this study were indicative of a significant difference between the rate of SBS and possibility of mixed-mode graft failure in groups 1-8. In other words, higher SBS rate was found to be associated with increased possibility of mixed-mode graft failure $(\mathrm{p}<0.005)$. The estimated rate of mixed-mode graft failure was $72.5 \%$ in this study. Furthermore, the possibility of mixed-mode graft failure was observed to increase with higher SBS rates in groups 9-16 ( $<<0.005)$, and the possibility of this graft failure mode was estimated at $80 \%$ in these sample groups.

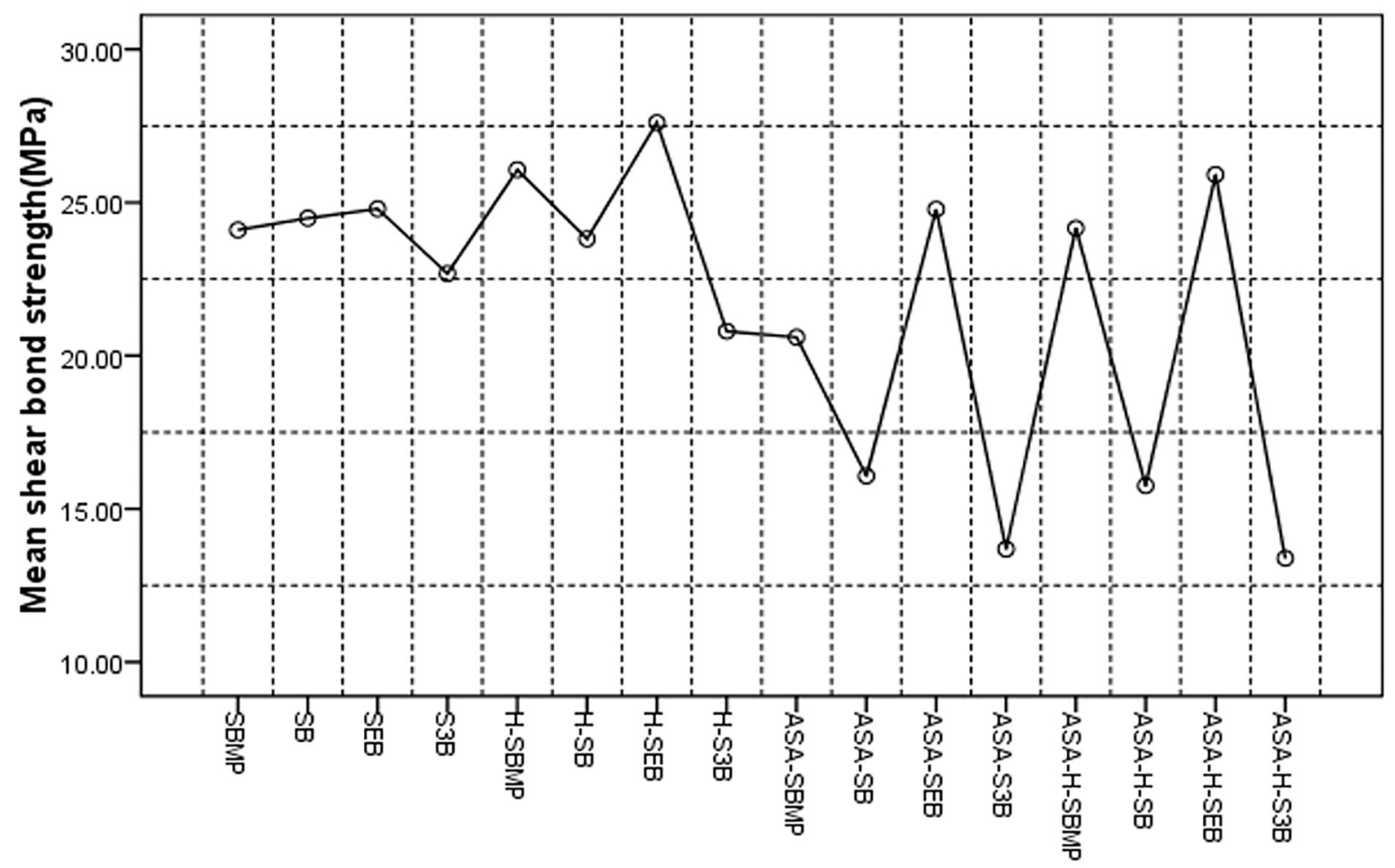

Figure 2. Mean of SBS (MPa) in different groups of total-etch and self-etch adhesive systems

\section{Discussion}

According to the results of the present study, SBS rate decreased in the teeth samples that were stored in artificial saliva for three months without the use of hydrophobic resin in all the adhesive systems. Furthermore, the SBS rate was observed to reduce in the samples with hydrophobic resin layer in all adhesive types, which was considered significant in SB and S3B adhesive systems. However, the SBS rate increased in samples without storage with a hydrophobic resin layer in the SBMP and SEB adhesive systems; this increase was significant in the SEB adhesive system only, while the SBS rate was reported to decrease in the SB and S3B adhesive systems. Use of hydrophobic resin in the SB and S3B adhesive systems was found to reduce the rate of SBS in samples that were not stored in artificial saliva. On the other hand, the SBS rate was observed to increase in the SBMP and SEB adhesive systems with the application of hydrophobic resin, which was only significant in the SBMB adhesive system. Several studies have confirmed the adverse effects of sample storage in water and aging on adhesive bond strength. For instance, findings of Shirai et al. regarding the effect of aging in one-step self-etch, two-etch, and rinse adhesive systems were in congruence with the results of the present study (10). According to a study by Burke et al., cured adhesive layers in one-step adhesives acted as a semi-permeable layer leading to the release of water from hydrated dentin into the adhesive bonding surface and composites; consequently, the osmotic release of the cured adhesive layer causes the bond strength to decrease (11). Another study in this regard showed that increased hydrophilic properties of the resins used in the structures of adhesives led to the reduction of tensile bond strength (12). In general, results of the mentioned study indicated that the rates of water absorption index and water solubility index mainly depend on hydrophilic adhesive compositions (13). Based on these findings, which have been confirmed by other studies (14, 15), it could be inferred that high rate of permeability in hydrophilic dental resins is a major issue affecting the mechanical stability of the resins, which ultimately leads to the reduction of the SBS rate. In another research in this 
regard, Van Landuyt et al. assessed the effect of the application of a hydrophobic bonding agent on bond strength. According to the findings, the addition of a preceding etching step could contribute to the bond strength of the enamel, while it had no effect on the bond strength of the dentin (9). This finding was in line with the results of other studies in this regard (16). Currently, two main methods are available to improve the bond strength of adhesives; one method involves the use of two or more layers of adhesive, and the other encompasses the use of an additional hydrophobic layer with the adhesives (8). According to the literature, bond strength is higher in increased solventfree adhesive systems, while the rate of leakage is likely to decrease with the use of additional layers (8). Another research in this regard suggested the use of two layers of self-etching primers to facilitate the bonding process (17); as such, the bond strength could improve with increased number of overlying layers in two all-in-one adhesive systems. According to the results of the current study, storage of samples in artificial saliva for three months without the use of margin bond reduced the SBS rate in all adhesive systems, while it significantly increased the SBS rate of the SBMP adhesive, and this increase was not considered significant in the SBS rate of the Clearfil SE bond. This finding was consistent with the results obtained by similar studies $(13,18-21)$. On the other hand, use of an additional layer of margin bond for the samples stored in artificial saliva significantly increased the SBS rates of the SBMP and SEB adhesive systems. In this regard, our findings indicated that use of margin bond could lead to the reduction of the SBS rate in all types of adhesives, which is in congruence with the results of similar studies (19, 20, 22-25). Researchers of the present study observed a significant increase in the SBS rate of the SBMP adhesive system, while this increase was insignificant in the SEB system, following the application of an additional layer of margin bond to the samples stored in artificial saliva. Furthermore, use of an additional layer of margin bond for the samples stored in artificial saliva caused a significant increase in the SBS rate of the SEB adhesive system, while this increase was considered insignificant in the SBMP adhesive system. However, the findings of Maleknejad et al. denoted that use of an additional layer of margin bond significantly increased the SBS rate of the SB and SEB adhesive systems (3). According to the results of the present study, the SBS rate of total-etch adhesives was higher compared to self-etch adhesives, while the lowest rate of SBS was observed in the S3B adhesive system. Higher SBS rate of total-etch adhesives compared to self-etch adhesives might be due to the influence of etchants and depth of the hybrid layer in total-etch adhesives. Furthermore, it is presumed that several dental tissues, especially the enamel, remain intact in self-etch adhesives due to simultaneous etching and priming. In other words, techniques of etching and rinsing of teeth are still considered remarkably effective in the strength and stability of bonds (26). According to the findings of the present study, the main cause of higher SBS rates in two-step self-etch adhesives compared to one-step adhesives is the differences in the ratios of the components of these adhesive systems. Twostep self-etch adhesives have a pure priming solution containing only functional monomers that dissolve in water. In a research by Abdallah et al., reduced SBS rates were observed in all self-etch adhesive systems, with the exception of SE, after aging (27). The hydrophilic nature of these resin compounds increases water absorption and resin-dentin permeability, leading to hydrolytic instability after aging (28); however, the SEB system is able to create a hybrid layer with lower sensitivity to aging. Moreover, high percentage of camphorquinone in this adhesive system was shown to enhance the degree of polymerization (29). In the current study, we suggested the use of an additional layer of hydrophobic resin, along with a two-step self-etch SEB adhesive in composite restorations. According to a study by Mine et al., Clearfil SE Bond has a higher SBS rate compared to other self-etch adhesive systems (30). However, the results of another study were indicative of no significant difference between one-step self-etch adhesives stored in water and other adhesive systems in this regard (31). This was inconsistent with the findings of the present study. On the other hand, findings of another study showed that the application of hydrophobic resin layer could improve microtensile bond strength of self-etch adhesives in dental tissues, especially the dentin (32).

Finally, the results of the current study were indicative of a direct relationship between mixed-mode graft failure and SBS rate. As such, graft failure is more likely to occur in the mixed form compared to adhesive graft failure in all experimental groups. This finding has been confirmed by similar studies $(18,28,33,34)$. In this regard, a number of studies have suggested that increased SBS may lead to the conversion of the graft failure from the adhesive type to mixed and cohesive types $(34,35)$, which is consistent with the results of the present study. The direct correlation between the mixed-mode graft failure and high SBS rate in the current research could be attributed to the high rate of intense mixing adhesives, composites, and dentin. It seems that further investigation is necessary regarding the effects of unfilled resin in self-etch and total-etch dentin adhesives on the SBS rate of composite to dentin. Furthermore, it is recommended that future studies evaluate the possible effects of ASA on the rate of SBS.

\section{Conclusions}

According to the results of this study, there was no significant difference in the mean of SBS rate between the first four experimental groups. However, a significant difference was observed between all types of adhesives in terms of 
the rate of SBS in experimental groups 5-8, 9-12 and 13-16. In addition, the highest and lowest SBS rates were reported in the SEB and S3B adhesive systems. The results of this study indicated that use of a hydrophobic resin layer could influence the SBS rate, while the mild SEB self-etch adhesive exhibited the most effective resin-dentine bond durability.

\section{Acknowledgments:}

The current study was internally funded by the School of Dentistry, Mashhad University of Medical Sciences. The authors thank Dr. Fatemeh Maleknejad and Dr. Mostafa Moazami for their help and comments.

\section{Conflict of Interest:}

There is no conflict of interest to be declared.

\section{Authors' contributions:}

All authors contributed to this project and article equally. All authors read and approved the final manuscript.

\section{References:}

1) Zimmerman RS, DiClemente RJ, Andrus JK, Hosein EN. Introduction to Global Health Promotion: Wiley. 2016.

2) Termeie D. Avoiding and Treating Dental Complications: Best Practices in Dentistry. Wiley; 2016.

3) Maleknejad F, Moazzami SM, Baradaran Nasseri E, Baradaran Nasseri E. Efficacy of hydrophobic layer on sealing ability of dentin adhesive systems in class V composite resin restorations. J Dental Res Dent Clin Dent Prospects. 2011; 5(1): 5-9. doi: 10.5681/joddd.2011.002. PMID: 23019500, PMCID: PMC3429989.

4) Yi YA, Ahn JS, Park YJ, Jun SH, Lee IB, Cho BH, et al. The effect of sandblasting and different primers on shear bond strength between yttria-tetragonal zirconia polycrystal ceramic and a self-adhesive resin cement. Oper Dent. 2015; 40(1): 63-71. doi: 10.2341/13-149-L. PMID: 25084110.

5) Rodrigues RF, Ramos CM, Francisconi PA, Borges AF. The shear bond strength of self-adhesive resin cements to dentin and enamel: An in vitro study. J Prosthet Dent. 2015; 113(3): 220-7. doi: 10.1016/j.prosdent.2014.08.008. PMID: 25444282.

6) Rosa WL, Piva E, Silva AF. Bond strength of universal adhesives: A systematic review and meta-analysis. J Dent. 2015; 43(7): 765-76. doi: 10.1016/j.jdent.2015.04.003. PMID: 25882585.

7) Saavedra G, Neisser MP, Sinhoreti MAC, Machado C. Evaluation of bond strength of denture teeth bonded to heat polymerized acrylic resin denture bases. Brazil J Oral Sci. 2015; 3(9): 458-64.

8) Takamizawa T, Barkmeier WW, Tsujimoto A, Berry TP, Watanabe H, Erickson RL, et al. Influence of different etching modes on bond strength and fatigue strength to dentin using universal adhesive systems. Dent Mater. 2016; 32(2): e9-21. doi: 10.1016/j.dental.2015.11.005. PMID: 26719131.

9) Van Landuyt K, Peumans M, De Munck J, Lambrechts P, Van Meerbeek B. Extension of a one-step selfetch adhesive into a multi-step adhesive. Dent Mater. 2006; 22(6): 533-44. doi: 10.1016/j.dental.2005.05.010.

10) Shirai K, De Munck J, Yoshida Y, Inoue S, Lambrechts P, Suzuki K, et al. Effect of cavity configuration and aging on the bonding effectiveness of six adhesives to dentin. Dent Mater. 2005; 21(2): 110-24. doi: 10.1016/j.dental.2004.01.003. PMID: 15681009 .

11) Burke FJ. What's new in dentine bonding? Self-etch adhesives. Dent Update. 2004; 31(10): 580-2, 4-6, 8-9. PMID: 15656072.

12) Yiu CK, King NM, Pashley DH, Suh BI, Carvalho RM, Carrilho MR, et al. Effect of resin hydrophilicity and water storage on resin strength. Biomaterials. 2004; 25(26): 5789-96. doi: 10.1016/j.biomaterials.2004.01.026. PMID: 15147825.

13) Reis AF, Giannini M, Pereira PN. Influence of water-storage time on the sorption and solubility behavior of current adhesives and primer/adhesive mixtures. Oper Dent. 2007; 32(1): 53-9. doi: 10.2341/06-13. PMID: 17288329.

14) Tay FR, Lai CN, Chersoni S, Pashley DH, Mak YF, Suppa P, et al. Osmotic blistering in enamel bonded with one-step self-etch adhesives. J Dent Res. 2004; 83(4): 290-5. doi: 10.1177/154405910408300404. PMID: 15044501.

15) Malacarne J, Carvalho RM, de Goes MF, Svizero N, Pashley DH, Tay FR, et al. Water sorption/solubility of dental adhesive resins. Dent Mater. 2006; 22(10): 973-80. doi: 10.1016/j.dental.2005.11.020. PMID: 16405987. 
16) Sadek FT, Pashley DH, Ferrari M, Tay FR. Tubular occlusion optimizes bonding of hydrophobic resins to dentin. J Dent Res. 2007; 86(6): 524-8. doi: 10.1177/154405910708600607. PMID: 17525351.

17) Altmann AS, Collares FM, Leitune VC, Samuel SM. The effect of antimicrobial agents on bond strength of orthodontic adhesives: a meta - analysis of in vitro studies. Orthod Craniofac Res. 2016; 19(1): 1-9. doi: 10.1111/ocr.12100. PMID: 26257400.

18) Toledano M, Osorio R, Osorio E, Aguilera FS, Yamauti M, Pashley DH, et al. Durability of resin-dentin bonds: effects of direct/indirect exposure and storage media. Dent Mater. 2007; 23(7): 885-92. doi: 10.1016/j.dental.2006.06.030. PMID: 16949659.

19) França FM, dos Santos AJ, Lovadino JR. Influence of air abrasion and long-term storage on the bond strength of self-etching adhesives to dentin. Oper Dent. 2007; 32(3): 217-24. doi: 10.2341/06-61. PMID: 17555172 .

20) Osorio R, Pisani-Proenca J, Erhardt MC, Osorio E, Aguilera FS, Tay FR, et al. Resistance of ten contemporary adhesives to resin-dentine bond degradation. J Dent. 2008; 36(2): 163-9. doi: 10.1016/j.jdent.2007.12.002. PMID: 18248872.

21) Foxton RM, Melo L, Stone DG, Pilecki P, Sherriff M, Watson TF. Long-term durability of one-step adhesive-composite systems to enamel and dentin. Oper Dent. 2008; 33(6): 651-7. doi: 10.2341/07-166.

22) $\mathrm{Yu}$ L, Wang X, Tian FC, Gao XJ. [Microtensile bond strengths of one-step self-etching adhesive systems]. Zhonghua kou qiang yi xue za zhi. 2007; 42(4): 240-1. PMID: 17683675.

23) Yazici AR, Celik C, Ozgünaltay G, Dayangaç B. Bond strength of different adhesive systems to dental hard tissues. Oper Dent. 2007; 32(2): 166-72. doi: 10.2341/06-49. PMID: 17427826.

24) De Munck J, Van Landuyt K, Peumans M, Poitevin A, Lambrechts P, Braem M, et al. A critical review of the durability of adhesion to tooth tissue: methods and results. J Dent Res. 2005; 84(2): 118-32. doi: 10.1177/154405910508400204. PMID: 15668328.

25) Proença JP, Polido M, Osorio E, Erhardt MC, Aguilera FS, García-Godoy F, et al. Dentin regional bond strength of self-etch and total-etch adhesive systems. Dent Mater. 2007; 23(12): 1542-8. doi: 10.1016/j.dental.2007.02.001. PMID: 17379296.

26) Inoue S, Vargas MA, Abe Y, Yoshida Y, Lambrechts P, Vanherle G, et al. Microtensile bond strength of eleven contemporary adhesives to dentin. J Adhes Dent. 2001; 3(3): 237-45. PMID: 11803711.

27) Abdalla AI, Feilzer AJ. Four-year water degradation of a total-etch and two self-etching adhesives bonded to dentin. J Dent. 2008; 36(8): 611-7. doi: 10.1016/j.jdent.2008.04.011. PMID: 18514996.

28) Toledano M, Osorio R, Albaladejo A, Aguilera FS, Tay FR, Ferrari M. Effect of cyclic loading on the microtensile bond strengths of total-etch and self-etch adhesives. Oper Dent. 2006; 31(1): 25-32. doi: 10.2341/04-161. PMID: 16536190.

29) Brackett WW, Ito S, Tay FR, Haisch LD, Pashley DH. Microtensile dentin bond strength of self-etching resins: Effect of a hydrophobic layer. Oper Dent. 2004; 30(6): 733-8. PMID: 16382596.

30) Mine A, De Munck J, Cardoso MV, Van Landuyt KL, Poitevin A, Kuboki T, et al. Bonding effectiveness of two contemporary self-etch adhesives to enamel and dentin. J Dent. 2009; 37(11): 872-83. doi: 10.1016/j.jdent.2009.06.020. PMID: 19683377.

31) Reis A, Moura K, Pellizzaro A, Dal-Bianco K, de Andrade AM, Loguercio AD, et al. Durability of enamel bonding using one-step self-etch systems on ground and unground enamel. Oper Dent. 2009; 34(2): 181-91. doi: 10.2341/08-58. PMID: 19363974.

32) Albuquerque M, Pegoraro M, Mattei G, Reis A, Loguercio AD. Effect of double-application or the application of a hydrophobic layer for improved efficacy of one-step self-etch systems in enamel and dentin. Oper Dent. 2008; 33(5): 564-70. doi: 10.2341/07-145. PMID: 18833863.

33) Ausiello P, Apicella A, Davidson CL. Effect of adhesive layer properties on stress distribution in composite restorations - a 3D finite element analysis. Dent Mater. 2002; 18(4): 295-303. PMID: 11992906.

34) Burrow MF, Kitasako Y, Thomas CD, Tagami J. Comparison of enamel and dentin microshear bond strengths of a two-step self-etching priming system with five all-in-one systems. Oper Dent. 2008; 33(4): 456-60. doi: 10.2341/07-125. PMID: 18666505.

35) Abu-Hanna A, Gordan VV. Evaluation of etching time on dentin bond strength using single bottle bonding systems. J Adhes Dent. 2004; 6(2): 105-10. PMID: 15293418. 